\title{
Cosmological parameters from supernova observations: A critical comparison of three data sets
}

\author{
T. R. Choudhury ${ }^{1}$ and T. Padmanabhan ${ }^{2}$ \\ 1 SISSA/ISAS, via Beirut 2-4, 34014 Trieste, Italy \\ e-mail: chou@sissa.it \\ 2 IUCAA, Ganeshkhind, Pune, 411 007, India \\ e-mail: nabhan@iucaa.ernet.in
}

Received 27 April 2004 / Accepted 15 September 2004

\begin{abstract}
We extend our previous analysis of cosmological supernova type Ia data (Padmanabhan \& Choudhury 2003) to include three recent compilation of data sets. Our analysis ignores the possible correlations and systematic effects present in the data and concentrates mostly on some key theoretical issues. Among the three data sets, the first set consists of 194 points obtained from various observations while the second discards some of the points from the first one because of large uncertainties and thus consists of 142 points. The third data set is obtained from the second by adding the latest 14 points observed through HST. A careful comparison of these different data sets help us to draw the following conclusions: (i) All the three data sets strongly rule out non-accelerating models. Interestingly, the first and the second data sets favour a closed universe; if $\Omega_{\mathrm{tot}} \equiv$ $\Omega_{\mathrm{m}}+\Omega_{\Lambda}$, then the probability of obtaining models with $\Omega_{\mathrm{tot}}>1$ is $\gtrsim 0.97$. Hence these data sets are in mild disagreement with the "concordance" flat model. However, this disagreement is reduced (the probability of obtaining models with $\Omega_{\text {tot }}>1$ being $\approx 0.9$ ) for the third data set, which includes the most recent points observed by HST around $1<z<1.6$. (ii) When the first data set is divided into two separate subsets consisting of low $(z<0.34)$ and high $(z>0.34)$ redshift supernova, it turns out that these two subsets, individually, admit non-accelerating models with zero dark energy because of different magnitude zero-point values for the different subsets. This can also be seen when the data is analysed while allowing for possibly different magnitude zero-points for the two redshift subsets. However, the non-accelerating models seem to be ruled out using only the low redshift data for the other two data sets, which have less uncertainties. (iii) We have also found that it is quite difficult to measure the evolution of the dark energy equation of state $w_{X}(z)$ though its present value can be constrained quite well. The best-fit value seems to mildly favour a dark energy component with current equation of state $w_{X}<-1$, thus opening the possibility of existence of more exotic forms of matter. However, the data is still consistent with the the standard cosmological constant at 99 per cent confidence level for $\Omega_{\mathrm{m}} \gtrsim 0.2$.
\end{abstract}

Key words. supernovae: general - cosmology: miscellaneous - cosmological parameters

\section{Introduction}

Current cosmological observations, particularly those of supernova type Ia, show a strong signature of the existence of a dark energy component with negative pressure (Riess et al. 1998; Perlmutter et al. 1999; Riess 2000). The most obvious candidate for this dark energy is the cosmological constant (with the equation of state $w_{X}=p / \rho=-1$ ), which, however, raises several theoretical difficulties (for reviews, see Sahni \& Starobinsky 2000; Peebles \& Ratra 2003; Padmanabhan 2003). This has led to models for dark energy component which evolves with time (Ratra \& Peebles 1988; Wetterich 1988; Ferreira \& Joyce 1998; Frieman et al. 1995; Brax \& Martin 1999; Brax \& Martin 2000; Urena-Lopez \& Matos 2000; Barreiro et al. 2000; Zlatev et al. 1999; Albrecht \& Skordis 2000; Bilic et al. 2002).
Currently, there is a tremendous amount of activity going on in trying to determine the equation of state $w_{X}(z)$ and other cosmological parameters from observations of high redshift type Ia supernova (Garnavich et al. 1998; Astier 2000; Saini et al. 2000; Wang \& Garnavich 2001; Weller \& Albrecht 2001; Goliath et al. 2001; Wang \& Lovelace 2001; Leibundgut 2001; Trentham 2001; Podariu et al. 2001; Corasaniti \& Copeland 2002; Kujat et al. 2002; Minty et al. 2002; Maor et al. 2002; Weller \& Albrecht 2002; Gerke \& Efstathiou 2002; Rowan-Robinson 2002; Linder \& Jenkins 2003; Padmanabhan \& Choudhury 2003; Visser 2004; Caresia, Matarrese, \& Moscardini 2004; Alcaniz 2004; Wang \& Mukherjee 2004; Novello et al. 2003; Knop et al. 2003; Zhu \& Fujimoto 2003; Dev et al. 2004; Gong et al. 2004; Gong \& Chen 2004; Gong \& Duan 2004; Gong 2004; Bertolami 2004; Wang \& Tegmark 2004; Chae et al. 2004; McInnes 2004; 
Szydlowski \& Czaja 2004; Bertolami et al. 2004; Lima \& Alcaniz 2004; Nesseris \& Perivolaropoulos 2004; Alam, Sahni, \& Starobinsky 2004; Zhu, Fujimoto, \& He 2004; Alcaniz \& Pires 2004). While there has been a considerable activity in this field, one should keep in mind that there are several theoretical degeneracies in the Friedmann model, which can limit the determination of $w_{X}(z)$. To understand this, note that the only non-trivial metric function in a Friedmann universe is the Hubble parameter $H(z)$ (besides the curvature of the spatial part of the metric), which is related to the total energy density in the universe. Hence, it is not possible to determine the energy densities of individual components of energy densities in the universe from any geometrical observation. For example, if we assume a flat universe, and further assume that the only energy densities present are those corresponding to the non-relativistic dust-like matter and dark energy, then we need to know $\Omega_{\mathrm{m}}$ of the dust-like matter and $H(z)$ to a very high accuracy in order to get a handle on $\Omega_{X}$ or $w_{X}$ of the dark energy. This can be a fairly strong degeneracy for determining $w_{X}(z)$ from observations.

Recently, we discussed certain questions related to the determination of the nature of dark energy component from observations of high redshift supernova in Padmanabhan \& Choudhury (2003, hereafter Paper I). In the above work, we reanalyzed the supernova data using very simple statistical tools in order to focus attention on some key issues. The analysis of the data were intentionally kept simple as we subscribe to the point of view that any result which cannot be revealed by a simple analysis of data, but arises through a more complex statistical procedure, is inherently suspect and a conclusion as important as the existence of dark energy with negative pressure should pass such a test. The key results of our previous analysis were:

- Even if the precise value of $\Omega_{X}$ or the equation of state $w_{X}(z)$ is known from observations, it is not possible to determine the nature (or, say, the Lagrangian) of the unknown dark energy source using only kinematical and geometrical measurements. For example, if one assumes that the dark energy arises from a scalar field, then it is possible to come up with scalar field Lagrangians of different forms leading to same $w_{X}(z)$. As an explicit example, we considered two Lagrangians, one corresponding to quintessence (Peebles \& Ratra 1988; Ratra \& Peebles 1988; Zlatev et al. 1999) and the other corresponding to the tachyonic scalar fields (Padmanabhan 2002; Padmanabhan \& Choudhury 2002; Frolov et al. 2002; Shiu \& Wasserman 2002; Gibbons 2002; Fairbairn \& Tytgat 2002; Mukohyama 2002; Feinstein 2002; Bagla et al. 2003). These two fields are quite different in terms of their intrinsic properties; however, it is possible to make both the Lagrangians produce a given $w_{X}(a)$ by choosing the potential functions in the corresponding Lagrangians (for explicit examples and forms of potential functions, see Padmanabhan (2002); Paper I).

- Although the full data set of supernova observations strongly rule out models without dark energy, the high and low redshift data sets, individually, admit non-accelerating models with zero dark energy. It is not surprising that the high redshift data is consistent with non-accelerating models as the universe is in its decelerating phase at those redshifts. On the other hand, though the acceleration of the universe is a low redshift phenomenon, the non-accelerating models could not be ruled out using low redshift data alone because of large errors. Given the small data set, any possible evolution in the absolute magnitude of the supernovae, if detected, might have allowed the data to be consistent with the non-accelerating models.

- We introduced two parameters, which can be obtained entirely from theory, to study the sensitivity of the luminosity distance on $w_{X}$. Using these two parameters, we argued that although one can determine the present value of $w_{X}$ accurately from the data, one cannot constrain the evolution of $w_{X}$. The situation is worse if we add the uncertainties in determining $\Omega_{\mathrm{m}}$.

All the above conclusions were obtained by analysing only 55 supernova data points from a very simple point of view. In recent times, data points from various sets of observations have been compiled taking into account the calibration errors and other uncertainties. This enables us to repeat our analysis for much larger data sets, and see how robust are the conclusions of Paper I with respect to the choice of the data points. In this paper, we will compare three such data sets, which differ in their selection criteria for data points and redshift range covered.

The structure of the paper is as follows: in the next section, we describe the three data sets used in this paper, and then analyse them for models with non-relativistic dust-like matter and cosmological constant. Some key points regarding the importance of low and high redshift data are discussed. In Sect. 3, we briefly discuss the constraints on the dark energy equation of state and its evolution. The results are summarized in Sect. 4. Finally, the effect of our extinction-based selection criterion on the determination of cosmological parameters is discussed in the appendix.

\section{Recent supernova data and their analysis}

We begin with a brief outline of the method of our analysis of the supernova data. The observations essentially measure the apparent magnitude $m$ of a supernova at peak brightness which, after correcting for galactic extinction and possible $\mathrm{K}$-correction, is related to the luminosity distance $d_{\mathrm{L}}$ of the supernova through

$m(z)=\mathcal{M}+5 \log _{10} Q(z)$,

where

$Q(z) \equiv \frac{H_{0}}{c} d_{\mathrm{L}}(z)$

and

$\mathcal{M}=M+5 \log _{10}\left(\frac{c / H_{0}}{1 \mathrm{Mpc}}\right)+25=M-5 \log _{10} h+42.38$.

The parameter $M$ is the absolute magnitude of the supernovae after correcting for supernova light curve width - luminosity correlation (Riess et al. 1996; Perlmutter et al. 1997; Phillips et al. 1999). After applying the above correction, $M$, and hence $\mathcal{M}$, is believed to be constant for all supernovae of type Ia. 
For our analysis, we consider three sets of data available in the literature at present. For completeness, we describe the data sets in detail:

(i) TONRY: in this data set we start with the 230 data points listed in Tonry et al. (2003) alongwith the 23 points from Barris et al. (2004). These data points are compiled and calibrated from a wide range of different observations. For obtaining the best-fit cosmological model from the data, one should keep in mind that the very low-redshift points might be affected by peculiar motions, thus making the measurement of the cosmological redshift uncertain; hence we consider only those points which have $z>0.01$. Further, since one is not sure about the host galaxy extinction $A_{\mathrm{V}}$, we do not consider points which have $A_{\mathrm{V}}>0.5$. The effect of this selection criterion based on the extinction, is discussed in the appendix. Thus for our final analysis, we are left with only 194 points (identical to what is used in Barris et al. 2004), which is more than thrice compared to what was used in Paper I.

The supernova data points in Tonry et al. (2003) and Barris et al. (2004) are listed in terms of the luminosity distance

$\mu_{1}(z) \equiv m(z)-\mathcal{M}_{\mathrm{obs}}(z)=5 \log _{10} Q_{\mathrm{obs}}(z)$,

alongwith the corresponding errors $\sigma_{\mu_{1}}(z)$. Note that the quantity $\mu_{1}(z)$ is obtained from observations by assuming some value of $\mathcal{M}$. This assumed value of $\mathcal{M}$ (denoted by $\mathcal{M}_{\text {obs }}$ in Eq. (4)) does not necessarily represent the "true" $\mathcal{M}$, and hence one has to keep it as a free parameter while fitting the data.

Any model of cosmology will predict the theoretical value $Q_{\text {th }}\left(z ; c_{\alpha}\right)$ with some undetermined parameters $c_{\alpha}$ (which may be, for example, $\Omega_{\mathrm{m}}, \Omega_{\Lambda}$ ). The best-fit model is obtained by minimizing the quantity

$\chi_{1}^{2}=\sum_{i=1}^{M}\left[\frac{\mu_{1}\left(z_{i}\right)-\mathcal{M}_{1}-5 \log _{10} Q_{\mathrm{th}}\left(z_{i} ; c_{\alpha}\right)}{\sigma_{\mu_{1}}\left(z_{i}\right)}\right]^{2}$

where

$\mathcal{M}_{1}=\mathcal{M}-\mathcal{M}_{\mathrm{obs}}$

is a free parameter representing the difference between the actual $\mathcal{M}$ and its assumed value $\mathcal{M}_{\text {obs }}$ in the data. To take into account the uncertainties arising because of peculiar motions at low redshifts, we add an uncertainty of $\Delta v=500 \mathrm{~km} \mathrm{~s}^{-1}$ to the distance error (Tonry et al. 2003), i.e.,

$\sigma_{\mu_{1}}^{2}(z) \rightarrow \sigma_{\mu_{1}}^{2}(z)+\left(\frac{5}{\ln 10} 10^{-0.2 \mu_{1}} \frac{\Delta v}{c}\right)^{2}$.

Note that this correction is most effective at low redshifts (i.e., for small values of $\mu_{1}$ ). The minimization of (5) is done with respect to the parameter $\mathcal{M}_{1}$ and the cosmological parameters $c_{\alpha}$.

(ii) RIESS(w/o HST): recently, Riess et al. (2004) have compiled a set of supernova data points from various sources with reduced calibration errors arising from systematics. In particular, they have discarded various points from the TONRY data set where the classification of the supernova was not certain or the photometry was incomplete - it is claimed that this has increased the reliability of the sample. The most reliable set of data, named as "gold", contain 142 points from previously published data, plus 14 points discovered recently using HST (Riess et al. 2004). Our second data set consists of 142 points from the above "gold" sample of (Riess et al. 2004), which does not include the latest HST data (hence the name RIESS(w/o HST)). Essentially, this data set is similar to the TONRY data set in terms of the covered redshift range, but is supposed to be more "reliable" in terms of calibration and other uncertainties.

We would like to mention here that the data points in (Riess et al. 2004) are given in terms of the distance modulus

$\mu_{2}(z) \equiv m(z)-M_{\mathrm{obs}}(z)$,

which differs from the previously defined quantity $\mu_{1}(z)$ in Eq. (4) by a constant factor. Consequently, the $\chi^{2}$ is calculated from

$\chi_{2}^{2}=\sum_{i=1}^{M}\left[\frac{\mu_{2}\left(z_{i}\right)-\mathcal{M}_{2}-5 \log _{10} Q_{\mathrm{th}}\left(z_{i} ; c_{\alpha}\right)}{\sigma_{\mu_{2}}\left(z_{i}\right)}\right]^{2}$

where

$\mathcal{M}_{2}=\mathcal{M}-M_{\text {obs }}$.

Note that the errors $\sigma_{\mu_{2}}\left(z_{i}\right)$ quoted in Riess et al. (2004) already take into account the effects of peculiar motions.

(iii) RIESS: our third data set consists of all the 156 points in the "gold" sample of (Riess et al. 2004), which includes the latest points observed by HST. The main difference of this set from the previous two is that this covers the previously unpopulated redshift range $1<z<1.6$.

Before starting our analysis, we would like to caution the reader about two very important points. First, the errors $\sigma_{m}(z)$ used above do not contain uncertainties because of systematics. Any rigorous statistical analysis of the supernova data for determining the cosmological parameters must take into account the systematic errors. The errors might arise because of calibration uncertainties, K-correction, Malmquist bias, gravitational lensing or the evolutionary effects (Goobar et al. 2002a,b; Linder \& Huterer 2003; Perlmutter \& Schmidt 2003; Caresia et al. 2004; Huterer et al. 2004; Linder 2004; Wang 2004; Kim et al. 2004). Including such errors into the analysis requires much involved analysis. Once these systematic errors are included, the errors on the cosmological parameter estimations might be higher than what will be reported in this paper. In this respect, we would also like to add that the data sets RIESS and RIESS(w/o HST) are supposed to reduce some of the systematic and calibration uncertainties in data.

Second, our simple frequentist analysis holds good only when the errors $\sigma_{m}(z)$ are Gaussian and uncorrelated. While considerable amount of analysis exist in the literature working with these approximations, there are various systematics because of which such approximations do not hold true. For example, the uncertainties in calibrating the data would surely introduce correlations in the errors (Kim et al. 2004). Similarly, uncertainties in the host galaxy extinction would introduce non-Gaussian asymmetric errors. Neglecting such effects might result in lower errors on the estimated values of the cosmological parameters. Note that the main thrust of our analysis is to 


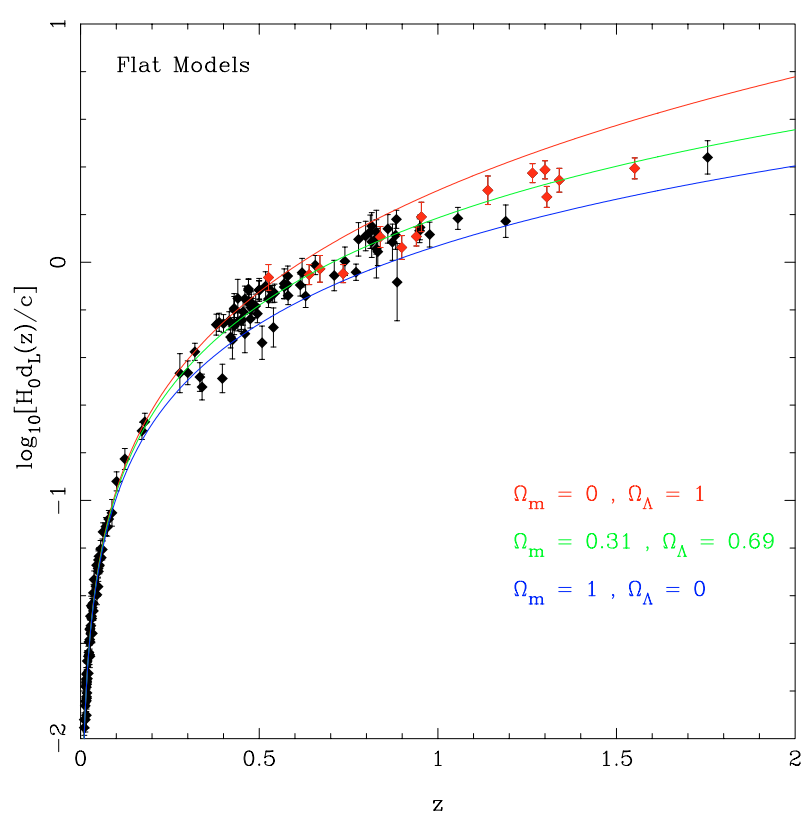

Fig. 1. Comparison between various flat models and the observational data. The observational data points, shown with error-bars, are obtained from the "gold" sample of Riess et al. (2004). The most recent points, obtained from HST, are shown in red. (This figure is available in color in electronic form.)

study some of the theoretical degeneracies inherent in any geometrical observations, in particular the supernova data, which are not adequately stressed elsewhere. Of course, this study can be complemented by other analyses which actually deal with quality and reliability of data, validity of error estimates, hidden correlations, nature of statistical analysis etc. All of these are important, but in order to make some key points we have attempted to restrict the domain of our exploration. Keeping this in mind, we believe that the simple (non-rigorous) $\chi^{2}$ analysis should be adequate.

Let us start our analysis with the flat models where $\Omega_{\mathrm{m}}+$ $\Omega_{\Lambda}=1$, which are currently favoured strongly by CMBR data (for recent WMAP results, see Spergel et al. 2003). Our simple analysis for the most recent RIESS data set, with two free parameters $\left(\Omega_{\mathrm{m}}, \mathcal{M}_{2}\right)$, gives a best-fit value of $\Omega_{\mathrm{m}}$ (after marginalizing over $\mathcal{M}_{2}$ ) to be $0.31 \pm 0.04$ (all the errors quoted in this paper are $1 \sigma$ ). This matches with the value $\Omega_{\mathrm{m}}=0.29_{-0.03}^{+0.05} \mathrm{ob}-$ tained by Riess et al. (2004). In comparison, the best-fit $\Omega_{\mathrm{m}}$ for flat models was found to be $0.31 \pm 0.08$ in Paper I - thus there is a clear improvement in the errors because of increase in the number of data points although the best-fit value does not change. The comparison between three flat models and the observational data from the RIESS data set is shown in in Fig. 1.

To see the accelerating phase of the universe more clearly, let us display the data as the phase portrait of the universe in the $\dot{a}-a$ plane. Though the procedure for doing this is described in Paper I (see also Daly \& Djorgovski 2003), we would like to discuss some aspects of the procedure in detail to emphasize a different approach we have used here in estimating the errors.
Each of the three sets of observational data used in this paper can be fitted by the function of simple form

$m_{\mathrm{fit}}(z)=a_{1}+5 \log _{10}\left[\frac{z\left(1+a_{2} z\right)}{1+a_{3} z}\right]$,

with $a_{1}, a_{2}, a_{3}$ being obtained by minimizing the $\chi^{2}$. We can then represent the luminosity distance obtained from the data by the function

$Q_{\text {fit }}(z)=10^{0.2\left[m_{\mathrm{fit}}(z)-\mathcal{M}\right]}$.

Note that one needs to fix the value of $\mathcal{M}$ to obtain the function $Q_{\text {fit }}(z)$. It is obvious, from the form of the fitting function (11) at low redshifts, that the parameter $a_{1}$ actually measures the quantity $\mathcal{M}$. It is then straightforward to obtain

$Q_{\text {fit }}(z)=\frac{z\left(1+a_{2} z\right)}{1+a_{3} z}$.

For flat models, it the Hubble parameter is related to $Q(z)$ by a simple relation - in this work we are interested in a related quantity

$H_{0}^{-1} \dot{a}(z)=\left[(1+z) \frac{\mathrm{d}}{\mathrm{d} z}\left\{\frac{Q(z)}{1+z}\right\}\right]^{-1}$

which will enable us to plot the data points in the $\dot{a}-a$ plane. Using the form of the fitting function, we can obtain the "fitted" $\dot{a}$ as:

$H_{0}^{-1} \dot{a}_{\mathrm{fit}}(z)=\frac{\left(1+a_{3} z\right)^{2}(1+z)}{1+2 a_{2} z+\left(a_{2}-a_{3}+a_{2} a_{3}\right) z^{2}}$.

To plot the individual supernova data points in the $\dot{a}-a$ plane, we first write $H_{0}^{-1} \dot{a}_{\text {fit }}$ as a function of $m_{\text {fit }}$ (which is trivially done by eliminating $z$ from Eqs. (11) and (15)). We then assume that the same relation can be applied to obtain the $\dot{a}$ corresponding to a particular measurement of $m$. Note that the relation between $\dot{a}$ and $m$ will involve the fitting parameters $a_{1}, a_{2}, a_{3}$, and hence is dependent on the fitting function.

The determination of the corresponding error-bars is a nontrivial exercise. In this paper, we obtain the error-bars using a Monte-Carlo realization technique, along the following lines: Given the observed values of $m(z)$ and $\sigma_{m}(z)$, we generate random realizations of the data set. Basically we randomly vary the magnitude of each supernova from a Gaussian distribution with dispersion $\sigma_{m}$ - each such set corresponds to one realization of the data set. Next, we fit each of the realization of the data sets with the fitting function (11), and obtain the set of three parameters $a_{1}, a_{2}, a_{3}$. Given the set of parameters $a_{1}, a_{2}, a_{3}$, we can obtain $\dot{a}$ for each $a$ (or equivalently, $z$ ). In this way we end up with different values of $\dot{a}$ for each supernova, each corresponding to one realization. Finally, we plot the distribution of $\dot{a}$ 's for each supernova, fit it with a Gaussian, and obtain the width of the Gaussian. This width is a possible candidate for the error in $\dot{a}$ for each supernova.

The data points, with error-bars, in the $\dot{a}-a$ plane are shown in Fig. 2 for all the three data sets. The solid curves plotted in Fig. 2 correspond to theoretical flat models with different $\Omega_{\mathrm{m}}$. In order to do any serious statistics with Fig. 2, one should 
RIESS

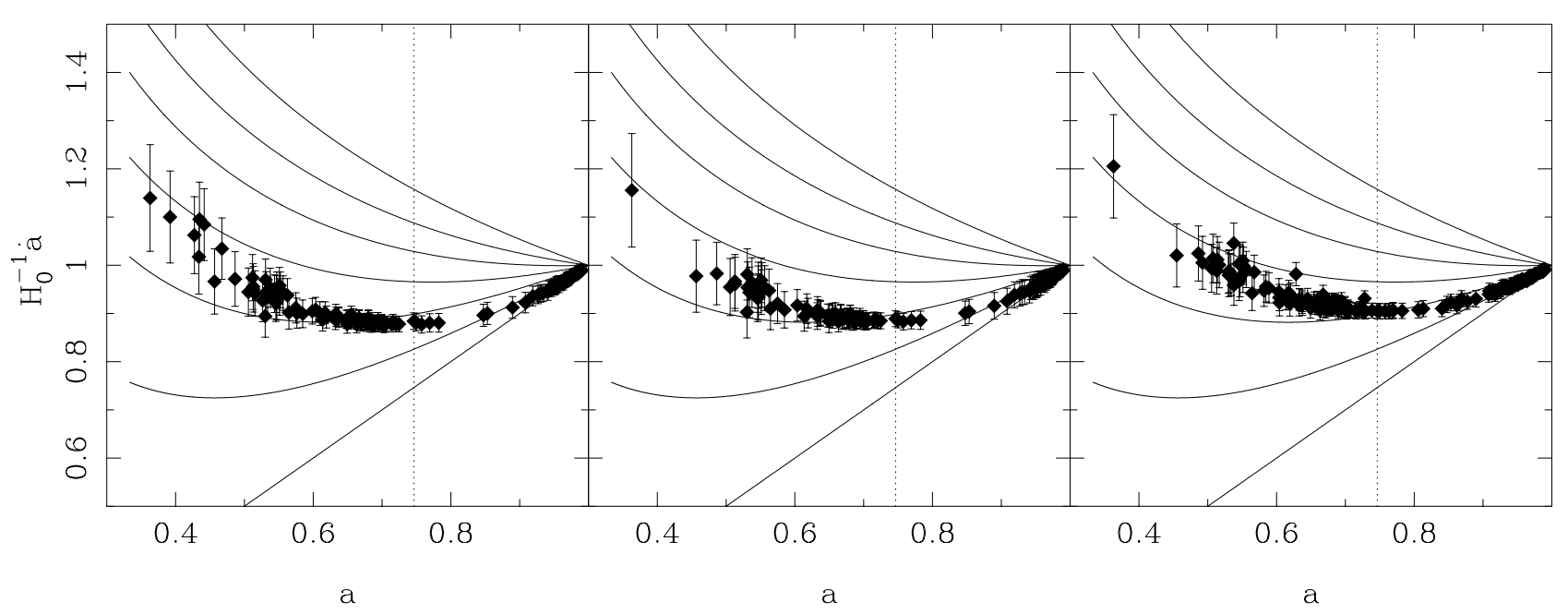

Fig. 2. The observed supernova data points in the $\dot{a}-a$ plane for flat models. The error bars for the data points are correlated (see text for detailed description). The solid curves, from bottom to top, are for flat cosmological models with $\Omega_{\mathrm{m}}=0.00,0.16,0.32,0.48,0.64,0.80,1.00$ respectively. The left, middle and right panels show data points for the data sets RIESS, RIESS(w/o HST) and TONRY respectively. The vertical dashed line shows the redshift $z=0.34$.

keep in mind that the errors for the data points in the figure are correlated.

It is obvious that the high redshift data alone cannot be used to establish the existence of a cosmological constant as the points having, say $a<0.75$, more or less, resemble a decelerating universe. In particular, one can use the freedom in the value of $\mathcal{M}$ to shift the data points vertically, and make them consistent with the non-accelerating $\operatorname{SCDM}$ model $\left(\Omega_{\mathrm{m}}=1\right.$, topmost curve). On the other hand, the low redshift data points show a clear, visual, sign of an accelerating universe at low redshifts. But to convert this visual impression into quantitative statistics is not easy since - as we said before - the errors at neighbouring points are correlated. We shall see later on, with correct statistical analysis, that it is, in general, quite difficult to rule out non-accelerating models using low redshift data alone, particularly when the uncertainties in the data are large.

Let us now make the above conclusions more quantitative by studying the confidence ellipses in the $\Omega_{\mathrm{m}}-\mathcal{M}_{1,2}$ plane, shown in Fig. 3, which should be compared with Fig. 4 of Paper I. For all the three rows, the left panels show the confidence regions using the full data sets. The confidence contours in the middle and right panels are obtained by repeating the best-fit analysis for the low redshift data set $(z<0.34)$ and high redshift data set $(z>0.34)$, respectively ${ }^{1}$. The three rows are for the three data sets respectively, as indicated in the figure itself.

When the supernova data is divided into low and high redshift subsets, the points to be noted are: (i) the best-fit value of $\mathcal{M}_{1,2}$ are substantially different for the two subsets

\footnotetext{
${ }^{1}$ One might notice that, in Paper I, we divided the high and lowredshift data points at $z=0.25$, whereas in this paper we divide them at $z=0.34$. The results of Paper I remain unchanged irrespective of whether the points are divided at $z=0.25$ or at $z=0.34$; this is because there were very few points between these redshifts.
}

(as indicated in the middle and right-hand panels of Fig. 3), irrespective of the data set used. The difference is most for the TONRY data set, comparatively less for the RIESS(w/o HST) data set and least for the RIESS data set. (ii) Because of the difference in the value of $\mathcal{M}_{1}$ for the TONRY data set, both the low and high redshift data subsets, when treated separately, are quite consistent with the SCDM model $\left(\Omega_{\mathrm{m}}=1\right)$. This indirectly stresses the importance of any evolutionary effects. If, for example, supernova at $z \gtrsim 0.34$ and supernova at $z \lesssim 0.34$ have different absolute luminosities because of some unknown effect, or if there is any systematics involved in estimating the magnitudes of the supernova, then the entire TONRY data set can be made consistent with the $\operatorname{SCDM}\left(\Omega_{\mathrm{m}}=1, \Omega_{\Lambda}=0\right)$ model. Comparing the bestfit values of $\mathcal{M}_{1}$ in the middle and right-hand panels in the lowest row of Fig. 3, one can see that a difference of about 0.5 mag in the absolute luminosities of the low and high-redshift supernova is sufficient to make the entire TONRY data set consistent with the SCDM model. This agrees with the point made in Paper I. (iii) However, the situation is markedly different for the other two data sets (RIESS(w/o HST) and RIESS), which are supposed to be more reliable than the TONRY data set. It turns out that because of less systematic errors, it is possible to rule out the SCDM model using the low redshift data alone as long as the absolute luminosities of supernovae do not evolve within the redshift range $z<0.34$. This is very important as it establishes the presence of the accelerating phase of the universe at low redshifts irrespective of the evolutionary effects. More reliable data sets at low redshifts will help in making this conclusion more robust.

Let us now consider the non-flat cosmologies where we have three free parameters, namely, $\Omega_{\mathrm{m}}, \Omega_{\Lambda}$ and $\mathcal{M}_{1,2}$. The confidence region ellipses in the $\Omega_{\mathrm{m}}-\Omega_{\Lambda}$ plane (after marginalizing over $\mathcal{M}_{1,2}$ ) are shown in Fig. 4 for the three data sets. 


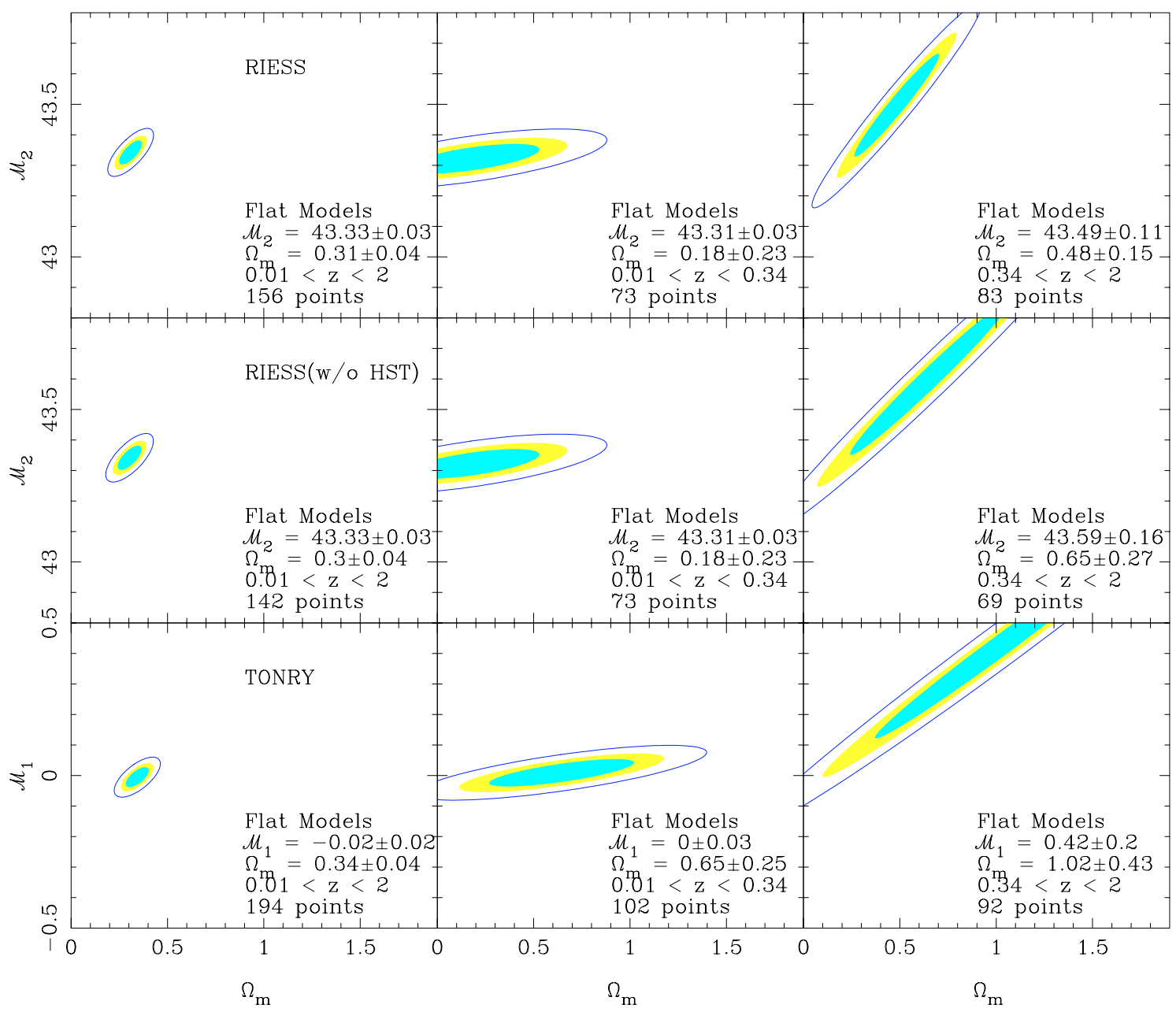

Fig. 3. Confidence region ellipses in the $\Omega_{\mathrm{m}}-\mathcal{M}_{1,2}$ plane for flat models with non-relativistic matter and a cosmological constant. The ellipses corresponding to the 68,90 and 99 per cent confidence regions are shown. The top, middle and bottom rows show data points for the data sets RIESS, RIESS(w/o HST) and TONRY respectively. In the left panels, all the data points in the data set are used. In the middle panel, data points with $z<0.34$ are used, while in the right panel, we have used data points with $z>0.34$. We have indicated the best-fit values of $\Omega_{\mathrm{m}}$ and $\mathcal{M}_{1,2}$ (with $1 \sigma$ errors).

The left panels, for all the three rows, give the confidence contours for the full data sets. One can compare the equivalent panel (a) of Fig. 5 in Paper I with the left panels of Fig. 4 and see that they are essentially similar. In the previous case the best-fit values for the full data set were given by $\Omega_{\mathrm{m}}=0.67 \pm 0.25, \Omega_{\Lambda}=1.24 \pm 0.34$, which agree, within allowed errors, with the best-fit values (indicated in the figure itself) for all the three data sets. The slanted shape of the probability ellipses in the left panels show that a particular linear combination of $\Omega_{\mathrm{m}}$ and $\Omega_{\Lambda}$ is selected out by these observations (which turns out to be $0.81 \Omega_{\mathrm{m}}-0.58 \Omega_{\Lambda}$ for the TONRY and RIESS(w/o HST) data sets, while it is $0.85 \Omega_{\mathrm{m}}-0.53 \Omega_{\Lambda}$ for the RIESS data set). This feature, of course, has nothing to do with supernova data and arises purely because the luminosity distance $Q$ depends strongly on a particular linear combination of $\Omega_{\mathrm{m}}$ and $\Omega_{\Lambda}$ (Goobar \& Perlmutter 1995). This point is illustrated by plotting the contour of constant luminosity distance, $Q(z)=$ constant in the left panels. The coincidence of this line (which roughly corresponds to $Q$ at a redshift in the middle of the data) with the probability ellipses indicates that it is the dependence of the luminosity distance on cosmological parameters which essentially determines the nature of this result. This aspect was discussed in detail in Paper I.

One disturbing aspect of all the three data sets (also noticed in the data sets right from the early days) is that the best-fit model favours a closed universe with $\Omega_{\mathrm{tot}} \equiv \Omega_{\mathrm{m}}+\Omega_{\Lambda}>1$. It is repeatedly argued that, due to the highly correlated nature of the probability contours (indicated by the very elongated ellipses in the left panels of Fig. 4), the best-fit value does not mean much. While this is true, one can certainly ask what is the probability distribution for $\Omega_{\text {tot }}$ if we marginalize over everything else. Interestingly we get $\Omega_{\text {tot }}=1.91 \pm 0.41$ for the TONRY data set, $\Omega_{\mathrm{tot}}=1.98 \pm 0.36$ for the RIESS(w/o HST) data set and $\Omega_{\text {tot }}=1.44 \pm 0.28$ for the RIESS data set. Alternatively, one can also compute the probability $\mathcal{P}\left(\Omega_{\mathrm{tot}}>1\right)$ of obtaining $\Omega_{\mathrm{tot}}>1$, which is found to be $\mathcal{P}\left(\Omega_{\mathrm{tot}}>1\right)=$ 0.97 for the TONRY data set, $\mathcal{P}\left(\Omega_{\mathrm{tot}}>1\right)=0.99$ for the RIESS(w/o HST) data set and $\mathcal{P}\left(\Omega_{\text {tot }}>1\right)=0.88$ for the RIESS data set. Although there is a general consensus that the "concordance" cosmological model has $\Omega_{\text {tot }}=1$, one 


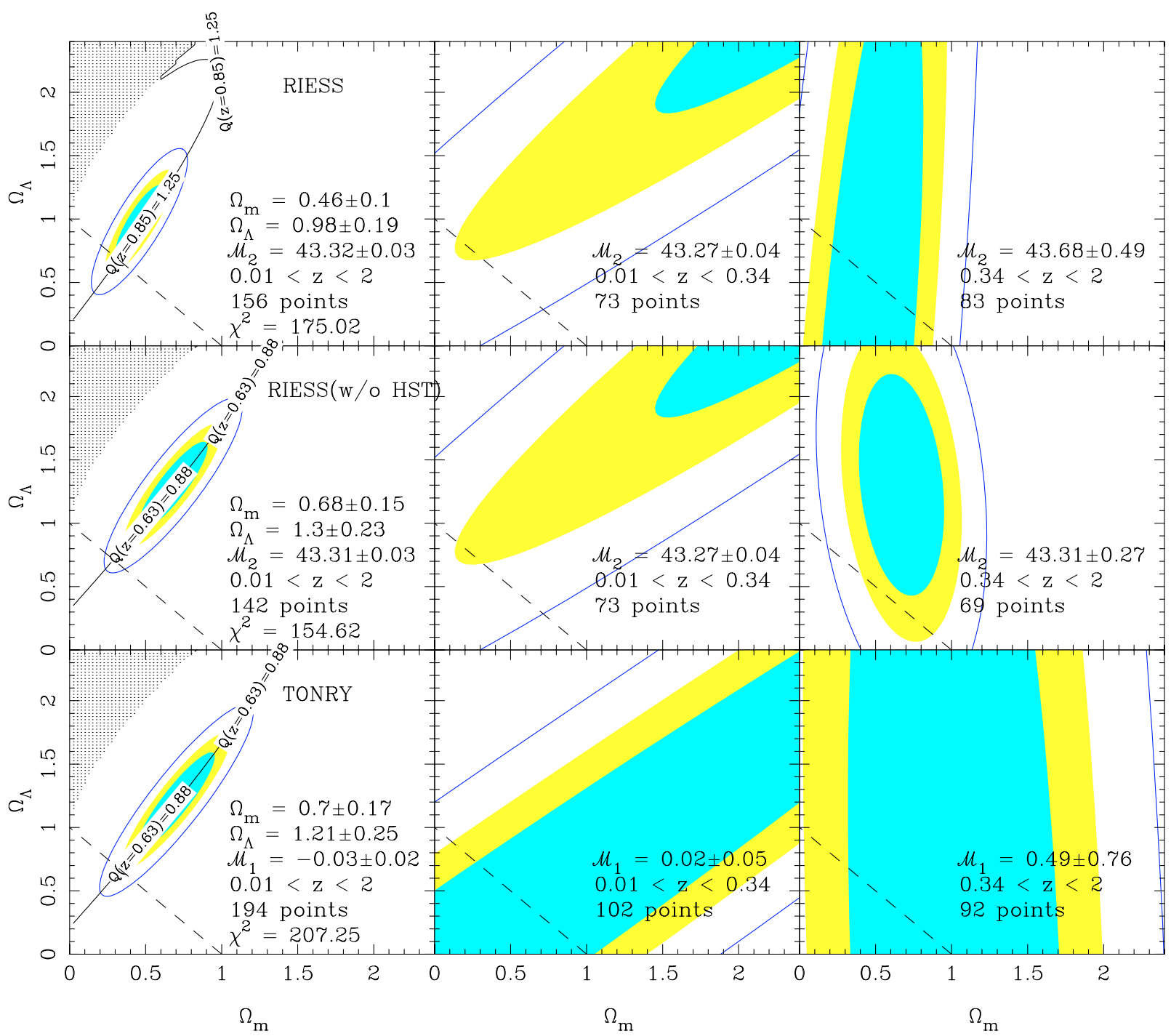

Fig. 4. Confidence region ellipses in the $\Omega_{\mathrm{m}}-\Omega_{\Lambda}$ plane for models with non-relativistic matter and a cosmological constant. The ellipses corresponding to the 68,90 and 99 per cent confidence regions are shown. The confidence regions are obtained after marginalizing over $\mathcal{M}_{1,2}$. The dashed line corresponds to the flat model $\left(\Omega_{\mathrm{m}}+\Omega_{\Lambda}=1\right)$. The unbroken slanted line corresponds to the contour of constant luminosity distance, $Q(z)=$ constant. The top, middle and bottom rows show data points for the data sets RIESS, RIESS(w/o HST) and TONRY respectively. In the left panels, all the data points in the data set are used. In the middle panel, data points with $z<0.34$ are used, while in the right panel, we have used data points with $z>0.34$. The values of the best-fit parameters, with $1 \sigma$ errors are indicated in the respective panels.

should keep in mind that as far as supernova data alone is concerned, it is highly probable that $\Omega_{\text {tot }}>1-$ in particular, the probability is quite high $(\gtrsim 0.97)$ when the recent HST data points are not included in the analysis. The presence of 14 new HST points at redshifts around 1 to 1.6 makes sure that the probability of obtaining $\Omega_{\mathrm{tot}}>1$ is somewhat lower $(<0.9)$.

Finally, we comment on the interplay between high and low redshift data for non-flat models. Just as in the case of the flat models, we divide the full data set into low $(z<0.34)$ and high $(z>0.34)$ redshift subsets, and repeat the best-fit analysis. The resulting confidence contours are shown in the middle and right panels of Fig. 4, which should be compared with panels (a) and (e) of Fig. 7 in Paper I. One can see that it is not possible to rule out the SCDM model using only high redshift data points when there are large uncertainties in $\mathcal{M}_{1,2}$, which agrees with what we concluded in Paper I. It is also clear that, like in
Paper I, the low redshift data for the TONRY data set cannot be used to discriminate between cosmological models effectively because of large errors on the data. However, the situation is quite different for the RIESS(w/o HST) and RIESS data sets. As we discussed before, the reduced uncertainties in these data sets have made it possible to rule out the SCDM model using low redshift data only. It is thus very important to have more data points at low redshifts (with less distance uncertainties) so as to conclude about the existence of accelerating phase of the universe, irrespective of evolutionary effects in absolute luminosities of supernovae.

We also note, as we did for flat models, that the best-fit value of $\mathcal{M}_{1,2}$ are substantially different for the two subsets (as indicated in the middle and right-hand panels of Fig. 4) with the difference being most for the TONRY data set and least for the RIESS data set. We can thus take our analysis one step 


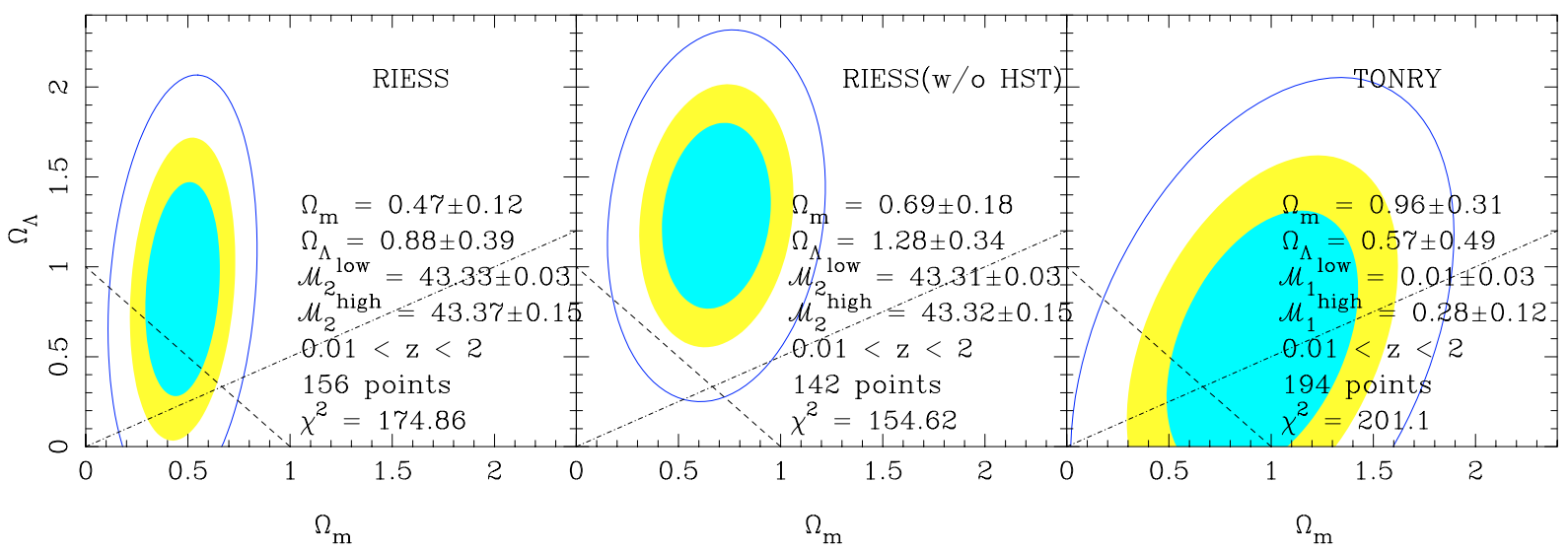

Fig. 5. Confidence region ellipses in the $\Omega_{\mathrm{m}}-\Omega_{\Lambda}$ plane for models with non-relativistic matter and a cosmological constant, allowing for possibly different $\mathcal{M}_{1,2}$ for the different redshift subsamples. It is assumed that supernovae at $z<0.34$ have $\mathcal{M}_{1,2}^{\text {low }}$, while those at $z>0.34$ have $\mathcal{M}_{1,2}^{\text {high }}$. The ellipses corresponding to the 68, 90 and 99 per cent confidence regions are shown. The confidence regions are obtained after marginalizing over $\mathcal{M}_{1,2}$. The dashed line corresponds to the flat model $\left(\Omega_{\mathrm{m}}+\Omega_{\Lambda}=1\right)$. The dot-dashed line denotes the models having zero deceleration at the present epoch (i.e., $q_{0}=0$ ), with the region below this line representing the non-accelerating models. The left, middle and right panels show data points for the data sets RIESS, RIESS(w/o HST) and TONRY respectively. The values of the best-fit parameters, with $1 \sigma$ errors are indicated in the respective panels.

further by fitting supernovae from all redshifts while allowing for possibly different $\mathcal{M}_{1,2}$ for the different redshift samples. To be precise, we assume that supernovae at lower redshifts $z<0.34$ have $\mathcal{M}_{1,2}^{\text {low }}$, while those at higher redshifts have $\mathcal{M}_{1,2}^{\text {high }}$. Given these, we can fit the data with four parameters and then marginalize over $\mathcal{M}_{1,2}^{\text {low }}$ and $\mathcal{M}_{1,2}^{\text {high }}$. The resulting confidence regions in the $\Omega_{\mathrm{m}}-\Omega_{\Lambda}$ plane are shown in Fig. 5 for the three data sets.

As is clear from the figure, one has quite different values for $\mathcal{M}_{1}^{\text {low }}$ and $\mathcal{M}_{1}^{\text {high }}$ for the TONRY data set, while the difference is lower for the other two data sets. This probably indicates that the difference in the values of $\mathcal{M}_{1}$ for different subsets for the TONRY data set arises from systematic errors, which are claimed to be reduced for the other two data sets. One requires more work, possibly a rigorous study using Monte-Carlo simulations, to understand this in detail. One should also note that the data is consistent with the nonaccelerating models at 68 and 99 percent confidence levels for the TONRY and RIESS data sets respectively, while they are ruled out for the RIESS(w/o HST) data set.

Before ending this section, let us explain a subtle point in determining $\Omega_{\mathrm{m}}$ and $\Omega_{\Lambda}$ from geometrical observations. As has been discussed in Paper I, the only non-trivial metric function in a Friedmann universe is the Hubble parameter $H(z)$ (besides the curvature of the spatial part of the metric), hence, it is not possible to determine the energy densities of individual components of energy densities in the universe from any geometrical observation. However, the analysis in this section might give the wrong impression that we have actually been able to determine both $\Omega_{\mathrm{m}}$ and $\Omega_{\Lambda}$ just from geometrical observations. The point to note that we have made a crucial additional assumption that the universe is dominated by non-relativistic matter and a cosmological constant, with known equations of state. Once this assumption about the equations of state is made, it allows us to determine the energy densities of the individual components. On the other hand, if, for example, we generalize the composition of the universe from a simple cosmological constant to a more general dark energy with unknown equation of state, it will turn out that the constraints will become much weaker. We shall take up this issue in the next section.

\section{Constraints on evolving dark energy}

As we have discussed in Paper I, the supernova data can be used for constraining the equation of state of the dark energy. In this section, we shall examine the possibility of constraining $w_{X}(z)$ by comparing theoretical models with supernova observations.

As done in Paper I, we parametrize the function $w_{X}(z)$ in terms of two parameters $w_{0}$ and $w_{1}$ :

$w_{X}(z)=w_{0}-w_{1}(a-1)=w_{0}+w_{1} \frac{z}{1+z}$,

and constrain these parameters from observations. We shall confine our analyses to flat models in this section (keeping in mind that the supernova data favours a universe with $\Omega_{\text {tot }}>1$ when $\left.w_{0}=-1, w_{1}=0\right)$.

If we assume $w_{X}$ does not evolve with time $\left(w_{1}=0\right)$, then a simple best-fit analysis for RIESS data set shows that for a flat model with $\Omega_{\mathrm{m}}=0.31$ and $\mathcal{M}_{2}=43.34$ (the best-fit parameters for flat models, obtained in the previous section), the best-fit value of $w_{0}$ is $-1.03 \pm 0.07$ (which is nothing but the conventional cosmological constant). The data, as before in Paper I, clearly rules out models with $w_{0}>-1 / 3$ at a high confidence level, thereby supporting the existence of a dark energy component with negative pressure.

One can extend the analysis to find the constraints in the $w_{0}-w_{1}$ plane. As before, we limit our analysis to a flat universe. Ideally, one should fit all the four parameters $\Omega_{\mathrm{m}}, \mathcal{M}_{1,2}, w_{0}, w_{1}$, and then marginalize over $\Omega_{\mathrm{m}}$ and $\mathcal{M}_{1,2}$ to obtain the constraints on $w_{X}$. However, if we put a uniform prior on $\Omega_{\mathrm{m}}$ in the whole range, then it turns out that it is impossible to get any sensible constraints on $w_{0}$ and $w_{1}$. Furthermore, we would like 


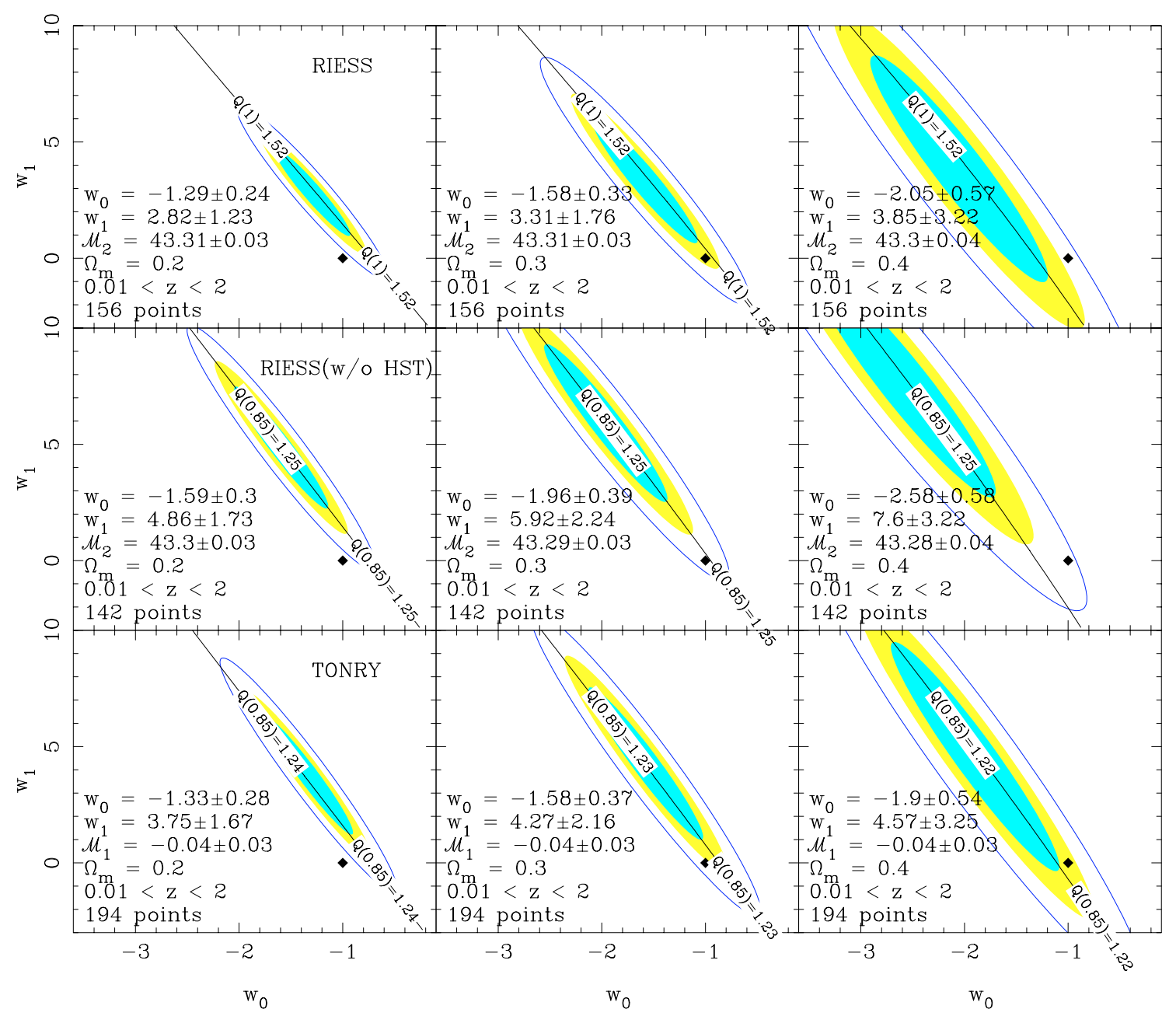

Fig. 6. Confidence region ellipses in the $w_{0}-w_{1}$ plane for flat models with a fixed value of $\Omega_{\mathrm{m}}$, as indicated in the frames. The confidence regions are obtained after marginalizing over $\mathcal{M}_{1,2}$. The ellipses corresponding to the 68,90 and 99 per cent confidence regions are shown. The square point denotes the equation of state for a universe with a non-evolving dark energy component (the cosmological constant). The unbroken slanted line corresponds to the contour of constant luminosity distance, $Q(z)=$ constant. The top, middle and bottom rows show data points for the data sets RIESS, RIESS(w/o HST) and TONRY respectively. The best-fit values of the fitted parameters $w_{0}$ and $w_{1}$ are indicated in the panels, alongwith the corresponding errors.

to present the results in such a manner so that one can see how the uncertainty in $\Omega_{\mathrm{m}}$ affects the constraints on $w_{X}$. Keeping this in mind, we fix the value of $\Omega_{\mathrm{m}}$ to $0.2,0.3$ and 0.4 (which are typical range of values determined by other observations, like the LSS surveys, and are independent of the nature of the dark energy; Pope et al. 2004; Tegmark et al. 2004b; Tegmark et al. 2004a), and marginalize only over $\mathcal{M}_{1,2}$.

The confidence contours for the three data sets are shown in Fig. 6, which can be compared with Fig. 8 of Paper I.

The square point denotes the equation of state for a universe with a non-evolving dark energy component (the cosmological constant). The main points revealed by this figure are: (i) the confidence contours are quite sensitive to the value of $\Omega_{\mathrm{m}}$ used, thus confirming the fact (which was mentioned in Paper I) that it is difficult to constrain $w_{X}$ with uncertainties in $\Omega_{\mathrm{m}}$. For example, in the TONRY data set, we see that nonaccelerating models with $w_{0}<-1 / 3$ are ruled out with a high degree of confidence for low values of $\Omega_{\mathrm{m}}$, while it is possible to accommodate them for $\Omega_{\mathrm{m}} \gtrsim 0.4$. We have elaborated this point in Paper I by studying the sensitivity of $Q(z)$ to $w_{0}$ and $w_{1}$ with varying $\Omega_{\mathrm{m}}$. (ii) The shape of the confidence contours clearly indicates that the data is not as sensitive to $w_{1}$ as compared to $w_{0}$. We stressed in Paper I that this has nothing to do with the supernova data as such. Essentially, the supernova observations measure $Q(z)$ and it turns out that $Q(z)$ is comparatively insensitive to $w_{1}$. (iii) The best-fit values for all the three data sets strongly favour models with $w_{0}<-1$, which indicate the possibility of exotic forms of energy densities - possibly scalar fields with negative kinetic energies (such models are explored, for example, in Caldwell 2002; Hannestad \& Mörtsell 2002; Carroll et al. 2003; Caldwell et al. 2003; Melchiorri et al. 2003; Singh et al. 2003; Johri 2004; Stefancic 2004; Sami \& Toporensky 2004; Li \& Hao 2004; Hao \& Li 2004; Szydlowski et al. 2004; Piao \& Zhang 2004). However, one should note that all the three data sets are still quite consistent with the standard cosmological constant $\left(w_{0}=-1, w_{1}=0\right)$ at 99 per cent confidence level for relatively higher values of $\Omega_{\mathrm{m}}$. One still requires data sets of better qualities to settle this issue. (iv) The 


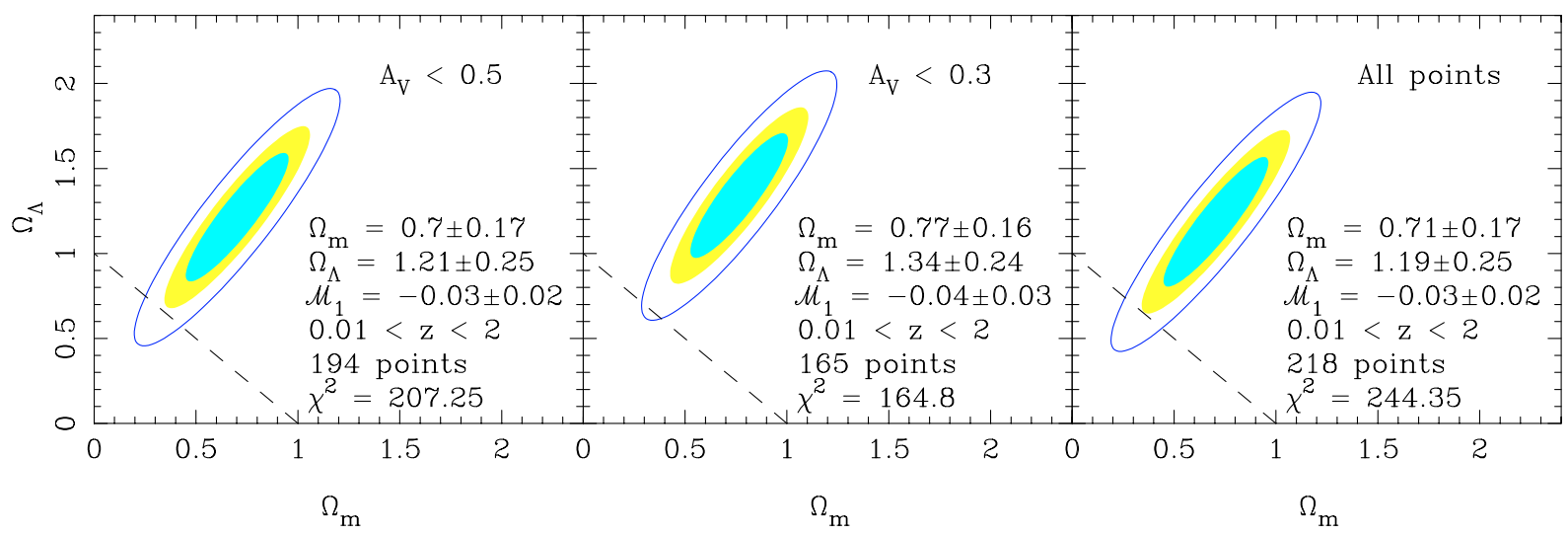

Fig. 7. Confidence region ellipses in the $\Omega_{\mathrm{m}}-\Omega_{\Lambda}$ plane for models with non-relativistic matter and a cosmological constant for different selection criteria based on extinction for the TONRY data set. The ellipses corresponding to the 68, 90 and 99 per cent confidence regions are shown. The confidence regions are obtained after marginalizing over $\mathcal{M}_{1}$. The dashed line corresponds to the flat model $\left(\Omega_{\mathrm{m}}+\Omega_{\Lambda}=1\right)$. The left panel shows results when only those points with $A_{\mathrm{V}}<0.5$ are included, the middle panel considers only points which have $A_{\mathrm{V}}<0.3$, while the right panel includes all the points irrespective of the value of $A_{\mathrm{V}}$. The values of the best-fit parameters, with $1 \sigma$ errors are indicated in the respective panels.

inclusion of the new HST data points (RIESS data set) have resulted in drastic decrease in the best-fit value of $w_{1}$ (from 5.92 to 3.31 for $\Omega_{\mathrm{m}}=0.3$ ), implying less rapid variation of $w_{X}(z)$.

\section{Discussion}

We have reanalyzed the supernova data with the currently available data points and constrained various parameters related to general cosmological models and dark energy. We would like to mention that our analysis ignores the effects of correlation and other systematics present in the data. The main aim of the work has been to focus on some important theoretical issues which are not adequately stressed in the literature. We have used three compiled and available data sets, which are called TONRY (194 points), RIESS(w/o HST) (142 points) and RIESS (156 points). The RIESS(w/o HST) is obtained from the TONRY data set by discarding points with large uncertainties and by reducing calibration errors, while the RIESS data set is obtained by adding the recent points from HST to the RIESS(w/o HST) set. The analysis is an extension to what was performed in Paper I with a small subset of data points. In particular, we have critically compared the estimated values of cosmological parameters from the three data sets. While the errors on the parameter estimation have come down significantly with all the data sets, we find that there some crucial differences between the data sets. We summarize the key results once more:

- It has been well known that the supernova data rule out the flat and open matter-dominated models with a high degree of confidence (Riess et al. 1998; Perlmutter et al. 1999; Riess 2000). However, for the TONRY and RIESS(w/o HST) data sets, we find that the data favours a model with $\Omega_{\text {tot }}>1$ (with probability $\gtrsim 0.97$ ) and is in mild disagreement with the "concordance" flat models with cosmological constant. This disagreement seem to be less (the probability of obtaining models with $\Omega_{\text {tot }}>1$ being $\approx 0.9$ ) for the RIESS data set, which includes the new HST points in the redshift range $1<z<1.6$,
- The supernova data on the whole rules out nonaccelerating models with very high confidence level. However, it is interesting to note that if we divide the TONRY data set into high and low redshift subsets, neither of the subsets are able to rule out the non-accelerating models. In particular, the low redshift data points are consistent with the nonaccelerating models because of large errors on the data. This keeps open the possibility that the evolutionary effects in the absolute luminosities of supernovae might make the entire data set consistent with SCDM model. The situation is quite different for the RIESS(w/o HST) and RIESS data sets, where points with large errors are discarded. The low redshift data alone seem to rule out the SCDM model with high degree of confidence. This means that unless the absolute luminosities of supernovae evolve rapidly with redshift, it might be difficult for the data set to be consistent with the SCDM model. In other words, the RIESS(w/o HST) and RIESS data sets establish the presence of the accelerating phase of the universe regardless of the evolutionary effects.

- The key issue regarding dark energy is to determine the evolution of its equation of state, $w_{X}$. We find that although one can constrain the current value of $w_{X}$ quite well, it is comparatively difficult to determine the evolution of $w_{X}$. The situation is further worsened when we take the uncertainties in $\Omega_{\mathrm{m}}$ into account.

- The supernova data mildly favours a dark energy equation of state with its present best-fit value less than -1 which will require more exotic forms of matter (possibly with negative kinetic energy). However, one should keep in mind that the data is still consistent with the standard cosmological constant at 99 per cent confidence level.

- The analysis of different subsamples of the supernova data set is important in determining the effect of evolution. In this work, we have taken the simple approach of dividing the data roughly around the epoch where the universe might have transited from a decelerating to an accelerating phase, and checked whether the data can be made consistent with the 
non-accelerating models. In future, it would be interesting to divide the data based on the nature of supernova searches. For example, one can divide the data into three redshift splits: $z<$ $0.1,0.2<z<0.8$ and $z>0.8$, which roughly correspond to supernovae discovered in shallow searches, ground-based deep searches, and space-based deep searches. It would be interesting to check the cosmological constraints with such a divide.

Acknowledgements. We thank Alex Kim for extensive comments which significantly improved the paper.

\section{Appendix: Effect of including supernovae with high extinction}

Since there is considerable uncertainty in determining the host extinction and reddening, we have considered only those supernova which have extinction $A_{\mathrm{V}}<0.5$ for the TONRY data set. It would be interesting to see how this selection criterion affects our determination of cosmological parameters. In particular, one should keep in mind that the high-redshift supernovae observed from the ground could have large uncertainty in their color and hence statistically will often have measured $A_{\mathrm{V}}>0.5$ even if they have no extinction.

To check how this affects the cosmological parameters, we concentrate on the cosmological models with non-relativistic matter and a cosmological constant, and find the constraints in the $\Omega_{\mathrm{m}}-\Omega_{\Lambda}$ plane. We consider three cases, namely, (i) the usual one where we exclude all the data points with $A_{\mathrm{V}}>0.5$, (ii) the one with a stricter selection criterion where we exclude points with $A_{\mathrm{V}}>0.3$ and finally (iii) we include all the points irrespective of the extinction. The results for the three cases are plotted in Fig. 7. It is clear from the figure that the exclusion of points based on their extinction have little effect on the determination of the cosmological parameters, at least for the TONRY data set. The cosmological parameters agree within $1 \sigma$ errors for the three different selection criteria.

\section{References}

Alam, U., Sahni, V., \& Starobinsky, A. A. 2004, JCAP, 0406, 008 Albrecht, A., \& Skordis, C. 2000, Phys. Rev. Lett., 84, 2076

Alcaniz, J. S. 2004, Phys. Rev. D, 69, 083521

Alcaniz, J. S., \& Pires, N. 2004, Phys. Rev. D, 70, 047303

Astier, P. 2000, Preprint [arXiv: astro-ph/0008306]

Bagla, J. S., Jassal, H. K., \& Padmanabhan, T. 2003, Phys. Rev. D, 67, 063504

Barreiro, T., Copeland, E. J., \& Nunes, N. J. 2000, Phys. Rev. D, 61, 127301

Barris, B. J., Tonry, J., Blondin, S., et al. 2004, ApJ, 602, 571

Bertolami, O. 2004, Preprint [arXiv: astro-ph/0403310]

Bertolami, O., Sen, A. A., Sen, S., \& Silva, P. T. 2004, MNRAS, 353, 329

Bilic, N., Tupper, G. B., \& Viollier, R. D. 2002, Phys. Lett. B, 535, 17

Brax, P., \& Martin, J. 1999, Phys. Lett. B, 468, 40

Brax, P., \& Martin, J. 2000, Phys. Rev., D, 61, 103502

Caldwell, R. R. 2002, Phys. Lett. B, 545, 23

Caldwell, R. R., Kamionkowski, M., \& Weinberg, N. N. 2003, Phys. Rev. Lett., 91, 071301

Caresia, P., Matarrese, S., \& Moscardini, L. 2004, ApJ, 605, 21
Carroll, S. M., Hoffman, M., \& Trodden, M. 2003, Phys. Rev. D, 68, 023509

Chae, K.-H., Chen, G., Ratra, B., \& Lee, D.-W. 2004, ApJ, 607, L71

Corasaniti, P. S., \& Copeland, E. J. 2002, Phys. Rev. D, 65, 043004

Daly, R. A., \& Djorgovski, S. G. 2003, ApJ, 597, 9

Dev, A., Jain, D., \& Alcaniz, J. S. 2004, A\&A, 417, 847

Fairbairn, M., \& Tytgat, M. H. G. 2002, Phys. Lett. B, 546, 1

Feinstein, A. 2002, Phys. Rev. D, 66, 063511

Ferreira, P. G., \& Joyce, M. 1998, Phys. Rev. D, 58, 023503

Frieman, J. A., Hill, C. T., Stebbins, A., \& Waga, I. 1995, Phys. Rev. Lett., 75, 2077

Frolov, A., Kofman, L., \& Starobinsky, A. A. 2002, Phys. Lett. B, 545, 8

Garnavich, P. M., Jha, S., Challis, P., et al. 1998, ApJ, 509, 74

Gerke, B. F., \& Efstathiou, G. 2002, MNRAS, 335, 33

Gibbons, G. W. 2002, Phys. Lett. B, 537, 1

Goliath, M., Amanullah, R., Astier, P., Goobar, A., \& Pain, R. 2001, Preprint [arXiv: astro-ph/0104009]

Gong, Y.-G. 2004, Preprint [arXiv: astro-ph/0401207]

Gong, Y.-g., \& Chen, X.-M. 2004, Preprint [arXiv: gr-qc/0402031]

Gong, Y.-G., Chen, X.-M., \& Duan, C.-K. 2004, Mod. Phys. Lett. A, 19,1933

Gong, Y.-G., \& Duan, C.-K. 2004, MNRAS, 352, 847

Goobar, A., Mörtsell, E., Amanullah, R., et al. 2002a, A\&A, 392, 757

Goobar, A., Mörtsell, E., Amanullah, R., \& Nugent, P. 2002b, A\&A, 393, 25

Goobar, A., \& Perlmutter, S. 1995, ApJ, 450, 14

Hannestad, S., \& Mörtsell, E. 2002, Phys. Rev. D, 66, 063508

Hao, J.-g., \& Li, X.-z. 2004, Preprint [arXiv: astro-ph/0404154]

Huterer, D., Kim, A., Krauss, L. M., \& Broderick, T. 2004, Preprint [arXiv: astro-ph/0402002]

Johri, V. B. 2004, Phys. Rev. D, 70, 041303

Kim, A. G., Linder, E. V., Miquel, R., \& Mostek, N. 2004, MNRAS, 347, 909

Knop, R. A., Aldering, G., Amanullah, R., et al. 2003, ApJ, 598, 102

Kujat, J., Linn, A. M., Scherrer, R. J., \& Weinberg, D. H. 2002, ApJ, 572,1

Leibundgut, B. 2001, ARA\&A, 39, 67

Li, X.-Z., \& Hao, J.-G. 2004, Phys. Rev. D, 69, 107303

Lima, J. A. S., \& Alcaniz, J. S. 2004, Phys. Lett. B, 600, 191

Linder, E. V. 2004, Preprint [arXiv: astro-ph/0406186]

Linder, E. V., \& Huterer, D. 2003, Phys. Rev. D, 67, 081303

Linder, E. V., \& Jenkins, A. 2003, MNRAS, 346, 573

Maor, I., Brustein, R., McMahon, J., \& Steinhardt, P. J. 2002, Phys. Rev. D, 65, 123003

McInnes, B. 2004, JHEP, 04, 036

Melchiorri, A., Mersini, L., Ödman, C. J., \& Trodden, M. 2003, Phys. Rev. D, 68, 043509

Minty, E. M., Heavens, A. F., \& Hawkins, M. R. S. 2002, MNRAS, 330, 378

Mukohyama, S. 2002, Phys. Rev. D, 66, 024009

Nesseris, S., \& Perivolaropoulos, L. 2004, Phys. Rev. D, 70, 043531

Novello, M., Perez Bergliaffa, S. E., \& Salim, J. 2003, Preprint [arXiv:astro-ph/0312093]

Padmanabhan, T. 2002, Phys. Rev. D, 66, 021301

Padmanabhan, T. 2003, Phys. Rept., 380, 235

Padmanabhan, T., \& Choudhury, T. R. 2002, Phys. Rev. D, 66, 081301

Padmanabhan, T., \& Choudhury, T. R. 2003, MNRAS, 344, 823 (Paper I)

Peebles, P. J., \& Ratra, B. 2003, Rev. Mod. Phys., 75, 559

Peebles, P. J. E., \& Ratra, B. 1988, ApJ, 325, L17

Perlmutter, S., Aldering, G., Goldhaber, G., et al. 1999, ApJ, 517, 565

Perlmutter, S., Gabi, S., Goldhaber, G., et al. 1997, ApJ, 483, 565 
Perlmutter, S., \& Schmidt, B. P. 2003, Lect. Notes Phys. (Berlin: Springer Verlag), 598, 195

Phillips, M. M., Lira, P., Suntzeff, N. B., et al. 1999, AJ, 118, 1766

Piao, Y.-S., \& Zhang, Y.-Z. 2004, Phys. Rev. D, 70, 063513

Podariu, S., Nugent, P., \& Ratra, B. 2001, ApJ, 553, 39

Pope, A. C., Matsubara, T., Szalay, A. S., et al. 2004, ApJ, 607, 655

Ratra, B., \& Peebles, P. J. E. 1988, Phys. Rev. D, 37, 3406

Riess, A. G. 2000, PASP, 112, 1284

Riess, A. G., Filippenko, A. V., Challis, P., et al. 1998, AJ, 116, 1009

Riess, A. G., Press, W. H., \& Kirshner, R. P. 1996, ApJ, 473, 88

Riess, A. G., Strolger, L., Tonry, J., et al. 2004, ApJ, 607, 665

Rowan-Robinson, M. 2002, MNRAS, 332, 352

Sahni, V., \& Starobinsky, A. A. 2000, Int. J. Mod. Phys. D, 9, 373

Saini, T. D., Raychaudhury, S., Sahni, V., \& Starobinsky, A. A. 2000, Phys. Rev. Lett., 85, 1162

Sami, M., \& Toporensky, A. 2004, Mod. Phys. Lett. A, 19, 1509

Shiu, G., \& Wasserman, I. 2002, Phys. Lett. B, 541, 6

Singh, P., Sami, M., \& Dadhich, N. 2003, Phys. Rev. D, 68, 023522

Spergel, D. N., Verde, L., Peiris, H. V., et al. 2003, ApJS, 148, 175

Stefancic, H. 2004, Eur. Phys. J. C, 36, 523

Szydlowski, M., \& Czaja, W. 2004, Preprint [arXiv: astro-ph/0402510]
Szydlowski, M., Czaja, W., \& Krawiec, A. 2004, Preprint [arXiv:astro-ph/0401293]

Tegmark, M., Blanton, M. R., Strauss, M. A., et al. 2004a, ApJ, 606, 702

Tegmark, M., Strauss, M. A., Blanton, M. R., et al. 2004b, Phys. Rev. D, 69, 103501

Tonry, J. L., Schmidt, B. P., Barris, B., et al. 2003, ApJ, 594, 1

Trentham, N. 2001, MNRAS, 326, 1328

Urena-Lopez, L. A., \& Matos, T. 2000, Phys. Rev. D, 62, 081302

Visser, M. 2004, Classical Quantum Gravity, 21, 2603

Wang, Y. 2004, Preprint [arXiv: astro-ph/0406635]

Wang, Y., \& Garnavich, P. M. 2001, ApJ, 552, 445

Wang, Y., \& Lovelace, G. 2001, ApJ, 562, L115

Wang, Y., \& Mukherjee, P. 2004, ApJ, 606, 654

Wang, Y., \& Tegmark, M. 2004, Phys. Rev. Lett., 92, 241302

Weller, J., \& Albrecht, A. 2001, Phys. Rev. Lett., 86, 1939

Weller, J., \& Albrecht, A. 2002, Phys. Rev. D, 65, 103512

Wetterich, C. 1988, Nucl. Phys., B302, 668

Zhu, Z.-H., \& Fujimoto, M.-K. 2003, ApJ, 585, 52

Zhu, Z.-H., Fujimoto, M.-K., \& He, X.-T. 2004, A\&A, 417, 833

Zlatev, I., Wang, L.-M., \& Steinhardt, P. J. 1999, Phys. Rev. Lett., 82, 896 